Conference Paper

\title{
Modelling and Thermal Analysis of Advanced Insulating Layer of Electronic Applications
}

Klarmann, S., Vagapov, Y. and Gotzig, H.

This is a paper presented at the 4th Int. Workshop on Thermal Investigations of ICs and Systems THERMINIC-2018, Stockholm, Sweden, 26-28 September 2018

Copyright of the author(s). Reproduced here with their permission and the permission of the conference organisers.

\section{Recommended citation:}

Klarmann, S., Vagapov, Y. and Gotzig, H. (2018), 'Modelling and thermal analysis of advanced insulating layer electronic applications'. In: Proc. 24th Int. Workshop on Thermal Investigations of ICs and Systems THERMINIC-2018, Stockholm, Sweden, 26-28 September 2018, pp. 1-4. 


\title{
Modelling and Thermal Analysis of Advanced Insulating Layer of Electronic Applications
}

\author{
Steffen Klarmann ${ }^{1}$, Yuriy Vagapov ${ }^{1}$, Heinrich Gotzig ${ }^{2}$ \\ ${ }^{1}$ Glyndwr University, School of Applied Sciences Computing and Engineering, Mold Road, Wrexham LL11 2AW, UK \\ ${ }^{2}$ Valeo S.A. Germany, Laiernstrasse 12, 74321 Bietigheim-Bissingen
}

\begin{abstract}
This paper proposes the replacement of a conventional Insulating-Metal-Substrate (IMS) dielectric layer with an electroconductive adhesive which is applied onto a treated aluminium heat sink. The adhesive based structure results in a lower thermal resistance of the system compared to IMS based on Aluminium. The model covers the investigation of two IMS

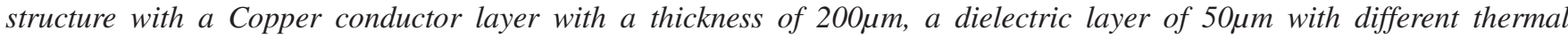

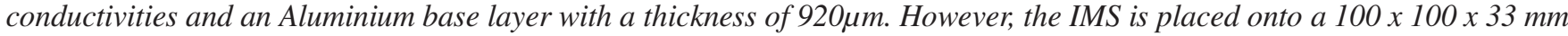
Aluminium heat sink. The investigated adhesive based structure consists of the same Aluminium heat sink treated with an

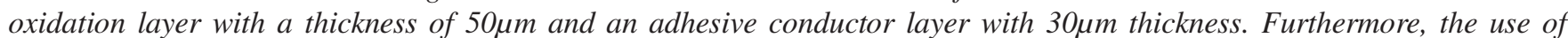
forced convection to reduce the junction temperature of all three models is also considered.
\end{abstract}

\section{Introduction}

The optical performance and reliability of LEDs are influenced by the junction temperature of the LED itself, therefore reduction of the dissipated heat is a crucial point in high-power electronic applications [1] [2]. Hence, in recent years, FR4 PCBs have been replaced by IMS PCBs in thermal applications to ensure a better heat dissipation and performance of the system [3]. However, several approaches to use FR4 technology in thermal applications have been investigated. By adding thermal vias below the heat dissipating electronic components, it is possible to reduce the thermal resistance and to improve the thermal performance of the system [4] [5]. A typical IMS PCB consists of a high thermal conductive material, like Aluminium or Copper. Based on this material, an insulating layer is applied, which ensures an electrical insulation between the metal core and the conductor layer on top of the IMS PCB [3] [4]. Since the thermal conductivity of the insulating layer is very low [6] [7], several approaches to improve the thermal conductivity already have been investigated [1] [3] [4] [8]. Hence, the insulating layer is the most critical part in an IMS PCB from a thermal point of view [3] [6]. The result of applying an anodizing process to Aluminium is an insulating surface layer. This electro nonconductive layer consists of hexagonal structures with a pore in the middle of each cell [9] [10]. These anodized coatings are usually applied to Aluminium to prevent the base material from environmental influence (e.g. corrosion, abrasion, etc.). By applying Nickel coating to the base material, it is possible to increase the thermal conductivity of the insulation layer. The common thickness of Nickel based coatings is varied from $2 \mu \mathrm{m}$ to $125 \mu \mathrm{m}$ [11]. Moreover, by applying Diamond coating, the thermal conductivity of the insulating layer results in a better thermal performance than anodizes based and Nickel based coatings [9]. By treat the heat sink (Copper or Aluminium) of a thermal system with a non-electro conductive insulating layer, it is possible to apply electro conductive adhesive onto the surface. However, it is possible to apply this adhesive in a defined structure, which can be used as tracks to provide the electronic connection between the components of the desired system. Hence, it is not required to implement a PCB onto such a system. Due to the reduction of the thermal resistance of the new adhesive based structure, the junction temperature of the LED is lower than using a regular IMS PCB. Nevertheless, the implementation of forced convection is also considered in this model to decrease the junction temperature.

A conventional IMS structure consists of a base layer with a high thermal conductive material (e.g. Aluminium or Copper). An insulation layer is applied between the base layer and the conductor layer on top of the IMS in order to provide a safe insulation between those two layers. The conductor layer consists of polymers and ceramics; this layer is required to prevent a short circuit from the base layer to the conductor layer. The electronic components are mounted onto the conductor layer. Therefore the conductor layer is made out of a high electro-conductive and high thermal-conductive material (e.g. Copper) to ensure a safe connection between the electronic components and efficient heat transfer.

This paper provides a comparison between IMS based PCBs and the proposed adhesive based PCB. The models of both PCBs are simulated to analyse the influence of natural and forced convection on the heat transfer.

\section{Mathematical Background}

In the presented model, natural convection, forced convection and conduction is applied. 


\subsection{Convection}

Natural convection occurs only in fluids and describes the thermal energy between them. In general, two different types of convection can occur - buoyancy driven (natural) convection and forced convection [12]. Newton's equation describes the natural convection as follows:

$$
Q=A h\left(T_{s}-T_{\infty}\right)
$$

where $Q$ is the heat transfer, $A$ the heat transfer area, $h$ the heat transfer coefficient. $T_{S}$ the surface temperature and $T_{\infty}$ the temperature of the fluid away from the surface.

\subsection{Conduction}

Conduction is the heat transfer between two media (e.g. solids, liquids, gas) by contact. The rate of heat in a one dimensional system, is described by Fourier's law as follows:

$$
q=k A \frac{\Delta T}{L}
$$

hence,

$$
Q=-k A \frac{d T}{d x}
$$

where $q$ is the heat transfer rate, $k$ the thermal conductivity, $A$ the cross section of the transfer area, $\Delta T$ the temperature difference, $L$ the length of the conduction path and $Q$ the heat flow rate in $\mathrm{x}$-direction.

\section{Thermal Model Description}

The ambient temperature applied to all simulations is set to $65^{\circ} \mathrm{C}$. The thermal LED dissipation power is $7 \mathrm{~W}$ transferred trough the heat sink with a size of $100 \times 100 \times 33$ mm made out of Aluminum. Additionally, the temperature of both the heat sink and the PCB is set to an initial value of $25^{\circ} \mathrm{C}$.

\subsection{IMS Model}

In the four simulated IMS models, the appropriate IMS PCB is assembled onto the Aluminum heat sink. The thickness of the conductor layer in each model is $200 \mu \mathrm{m}$, whereas the thermal conductivity of the conductor layer is $385 \mathrm{Wm}^{-1} \mathrm{~K}^{-1}$. Moreover, in the first and second model the thickness of the dielectric layer is $50 \mu \mathrm{m}$ and the thermal conductivity is $3 \mathrm{Wm}^{-1} \mathrm{~K}^{-1}$. However, the thermal conductivity of the third and fourth model is $5 \mathrm{Wm}^{-1} \mathrm{~K}^{-1}$, whereas the thickness in both models is $50 \mu \mathrm{m}$. Furthermore, the thickness of the base layer in all models is $920 \mu \mathrm{m}$ with a thermal conductivity of $138 \mathrm{Wm}^{-1} \mathrm{~K}^{-1}$. Table 1 shows the data of the simulated IMS Cauer model. It can be seen, that the thermal capacitance of the junction, solder and the dielectric layer is negligible compared to the thermal capacitance of the conductor, core and heat sink. Hence, Figure 1 illustrates the Cauer model of the IMS $\mathrm{PCB}$, where $R_{t h 1}$ is the thermal resistance between junction and solder, the thermal resistance between solder and conductor is describes as $R_{t h 2}, R_{t h 3}$ is the thermal resistance between conductor layer and dielectric layer, $R_{t h 4}$ is the thermal resistance between dielectric layer and core, $R_{t h 5}$ is the thermal resistance between core and heat sink, $R_{t h 6}$ is the thermal resistance between heat sink and ambient temperature, $T_{1}$ is the junction temperature, since $T_{2}$ is the ambient temperature. Furthermore, $C_{t h 3}$ is the thermal capacitance of the conductor, $C_{t h 5}$ is the thermal capacitance of the core and $C_{t h 6}$ is the thermal capacitance of the heat sink. Table 2 shows an overview of the IMS models, whereas Figure 2 illustrates the cross section of the simulated IMS structure. Furthermore for the second and forth model, a forced convection with $2 \mathrm{~ms}^{-1}$ is simulated.

\section{Table 1: IMS Cauer Model Data}

\begin{tabular}{|c|c|c|c|c|}
\hline Layer & $t$ & $R_{t h}$ & $C_{t h}$ & $\lambda$ \\
\hline & $\mu m$ & $K W^{l}$ & $J K^{-1}$ & $W m^{-l} K^{-l}$ \\
\hline Junction & 250 & 0.43 & $\mathbf{3 1 . 4} \cdot \mathbf{1 0}^{-\mathbf{6}}$ & 385 \\
\hline Solder & 250 & 2.87 & $\mathbf{1 0 . 9 5} \cdot \mathbf{1 0}^{-\mathbf{6}}$ & 60 \\
\hline Conductor & 200 & $\mathbf{0 . 2 8} \cdot \mathbf{1 0}^{-\mathbf{3}}$ & 1.2 & 385 \\
\hline Dielectric & 50 & $\mathbf{9 . 2 3} \cdot \mathbf{1 0}^{-3}$ & $\mathbf{0 . 2 2} \cdot \mathbf{1 0}^{-\mathbf{6}}$ & 3 \\
\hline Dielectric & 50 & $\mathbf{5 . 5 4} \cdot \mathbf{1 0}^{-\mathbf{3}}$ & $\mathbf{0 . 2 2} \cdot \mathbf{1 0}^{-\mathbf{6}}$ & 5 \\
\hline Core & 920 & $\mathbf{1 . 3 2} \cdot \mathbf{1 0}^{-\mathbf{3}}$ & 4.07 & 385 \\
\hline Heat Sink & 33,000 & $\mathbf{1 7 . 1 8} \cdot \mathbf{1 0}^{-\mathbf{3}}$ & 810 & 192 \\
\hline
\end{tabular}

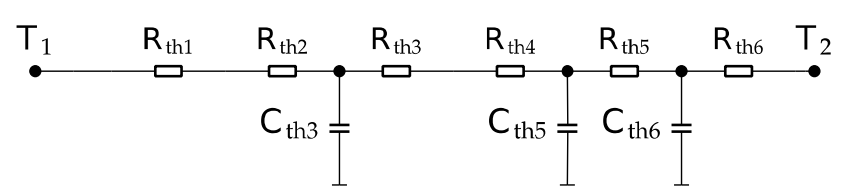

Figure 1: Cauer Model of Simulated IMS Thermal Resistance

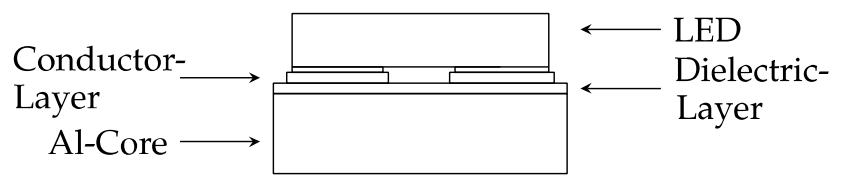

Figure 2: LED soldered on IMS PCB with the conductor layer assembled on the dielectric which is mounted on the Al-Core.

\subsection{Adhesive Based Model}

The Aluminum base in the two simulated adhesive based models is anodized. This anodizing results in a honeycomb structured surface of the Aluminum, which is electrically insulating. The simulated thickness of this anodized layer is $50 \mu \mathrm{m}$, whereas the thermal conductivity is simulated with $13.8 \mathrm{Wm}^{-1} \mathrm{~K}^{-1}$. By applying electro conductive adhesive to this insulating layer, it is possible to print tracks to the surface of the Aluminum. Therefore, the adhesive layer is simulated with a thickness of $30 \mu \mathrm{m}$ and a thermal conductivity of $5 \mathrm{Wm}^{-1} \mathrm{~K}^{-1}$. 
Table 2: IMS Model Overview

\begin{tabular}{|c|c|c|c|c|c|c|c|}
\hline No. & \multicolumn{2}{|c|}{ Conductor } & \multicolumn{2}{|c|}{ Dielectric } & \multicolumn{2}{c|}{ Base } & $\begin{array}{c}\text { Convec } \\
\text { tion }\end{array}$ \\
\hline & $\mathrm{t}$ & $\lambda_{\mathrm{c}}$ & $\mathrm{t}$ & $\lambda_{\mathrm{d}}$ & $\mathrm{t}$ & $\lambda_{\mathrm{b}}$ & \\
\hline & $\mu \mathrm{m}$ & $\mathrm{Wm}^{-1} \mathrm{~K}^{-1}$ & $\mu \mathrm{m}$ & $\mathrm{Wm}^{-1} \mathrm{~K}^{-1}$ & $\mu \mathrm{m}$ & $\mathrm{Wm}^{-1} \mathrm{~K}^{-1}$ & $\mathrm{~ms}^{-1}$ \\
\hline 1 & 200 & 385 & 50 & 3 & 920 & 138 & 0 \\
\hline 2 & 200 & 385 & 50 & 3 & 920 & 138 & 2 \\
\hline 3 & 200 & 385 & 50 & 5 & 920 & 138 & 0 \\
\hline 4 & 200 & 385 & 50 & 5 & 920 & 138 & 2 \\
\hline
\end{tabular}

Table 3 shows the data of the simulated adhesive Cauer model. It is shown that the thermal capacitance of the junction, as well as the adhesive layer can be negligible compared to the heat sink. Furthermore, Figure 3 depicts the simulated thermal resistance circuit of the adhesive based model, where $R_{t h 1}$ is the thermal resistance between junction and adhesive, the thermal resistance between adhesive and heat sink is described by $R_{t h 2}, R_{t h 3}$ is the thermal resistance between heat sink and ambient, $T_{1}$ is the junction temperature of the LED, whereas $T_{2}$ is the ambient temperature. In addition, $C_{t h 3}$ represents the thermal capacitance of the heat sink. Furthermore, the sixth model is simulated with a forced convection of $2 \mathrm{~ms}^{-1}$. Table 4 shows the overview of the simulated adhesive based models. It can be seen, that two models representing natural and forced convections are investigated. In addition, Figure 4 shows the cross-section of the simulated adhesive based model.

\section{Table 3: Adhesive Cauer Model Data}

\begin{tabular}{|c|c|c|c|c|}
\hline Layer & $\mathrm{t}$ & $\mathrm{R}_{\mathrm{th}}$ & $\mathrm{C}_{\mathrm{th}}$ & $\lambda$ \\
\hline & $\mu \mathrm{m}$ & $\mathrm{KW}^{-1}$ & $\mathrm{JK}^{-1}$ & $\mathrm{Wm}^{-1} \mathrm{~K}^{-1}$ \\
\hline Junction & 250 & 0.43 & $31.4 \cdot 10^{-6}$ & 385 \\
\hline Adhesive & 60 & 0.97 & $4.00 \cdot 10^{-6}$ & 13.5 \\
\hline Heat Sink & 33,000 & $17.18 \cdot 10^{-3}$ & 810 & 192 \\
\hline
\end{tabular}

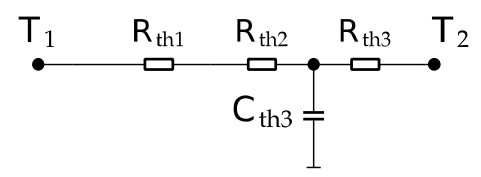

Figure 3: Cauer Model of Simulated Adhesive Bases PCB Thermal Resistance

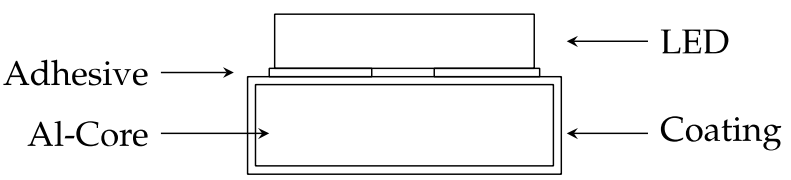

Figure 4: LED assembled onto the adhesive layer, which is applied to the coated Al-Core
Table 4: Adhesive Model Overview

\begin{tabular}{|c|c|c|c|c|c|c|c|}
\hline No. & \multicolumn{2}{|c|}{ Adhesive } & \multicolumn{2}{c|}{ Coating } & \multicolumn{2}{c|}{ Base } & $\begin{array}{c}\text { Convec } \\
\text { tion }\end{array}$ \\
\hline & $\mathrm{t}$ & $\lambda_{\mathrm{c}}$ & $\mathrm{t}$ & $\lambda_{\mathrm{d}}$ & $\mathrm{t}$ & $\lambda_{\mathrm{b}}$ & \\
\hline & $\mu \mathrm{m}$ & $\mathrm{Wm}^{-1} \mathrm{~K}^{-1}$ & $\mu \mathrm{m}$ & $\mathrm{Wm}^{-1} \mathrm{~K}^{-1}$ & $\mu \mathrm{m}$ & $\mathrm{Wm}^{-1} \mathrm{~K}^{-1}$ & $\mathrm{~ms}^{-1}$ \\
\hline 5 & 30 & 5 & 50 & 13.8 & 920 & 138 & 0 \\
\hline 6 & 30 & 5 & 50 & 13.8 & 920 & 138 & 2 \\
\hline
\end{tabular}

\section{Simulation Results}

\subsection{Natural Convection Model}

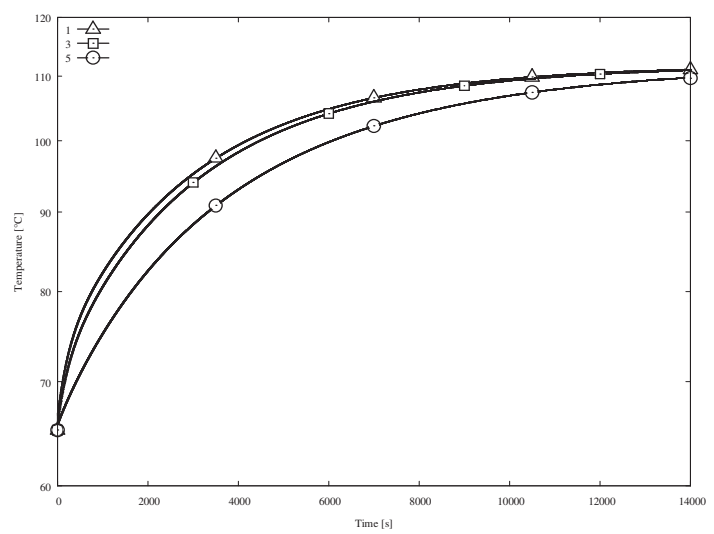

Figure 5: Natural Convection Simulation Result

Figure 5 shows the junction temperature of the LED with the simulated PCB models under natural convection. It can be seen, that increasing the thermal conductivity of the dielectric layer from $3 \mathrm{Wm}^{-1} \mathrm{~K}^{-1}$ to $5 \mathrm{Wm}^{-1} \mathrm{~K}^{-1}$ results in a reduced junction temperature of the LED. Nevertheless, the use of the adhesive based PCB will result in the lowest junction temperature. The steady-state junction temperature for the first model is $111.1^{\circ} \mathrm{C}$, for the third model $110.9^{\circ} \mathrm{C}$, however for the fifth model the junction temperature is $109.7^{\circ} \mathrm{C}$

\subsection{Forced Convection Model}

The junction temperature of the forced convection model is illustrated in Figure 6. It can be seen that the steady-state junction temperature for the second model is $105.9^{\circ} \mathrm{C}$, for the fourth model $105.8^{\circ} \mathrm{C}$, furthermore for the sixth model $104.8^{\circ} \mathrm{C}$

\subsection{Model Comparison}

Table 5 shows the junction temperatures of the natural convection models at steady-state condition. It can be seen, that the change of the dielectric conductivity from $3 \mathrm{Wm}^{-1} \mathrm{~K}^{-1}$ to $5 \mathrm{Wm}^{-1} \mathrm{~K}^{-1}$ results in a junction temperature, which is $0.2^{\circ} \mathrm{C}$ lower. Furthermore, using adhesive based model, the steady-state junction temperature is $1.4^{\circ} \mathrm{C}$ lower compared to the first IMS model. 


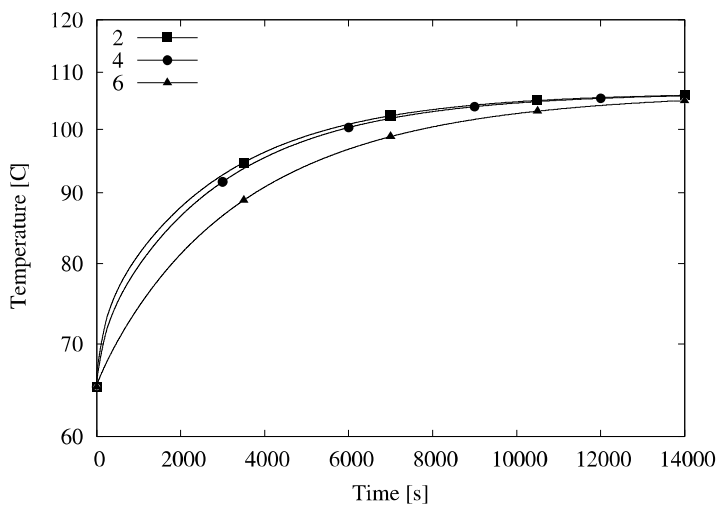

Figure 6: Forced Convection Simulation Result

Table 5: Natural Convection Junction Temperature Results

\begin{tabular}{|c|c|}
\hline No. & $\mathrm{T}_{\mathrm{j}}$ \\
\hline & ${ }^{\circ} \mathrm{C}$ \\
\hline 1 & 111.1 \\
\hline 3 & 110.9 \\
\hline 5 & 109.7 \\
\hline
\end{tabular}

Additionally, Table 6 illustrates the steady-state junciton temperatures of forced convection models. By applying a forced convection of $2 \mathrm{~ms}^{-1}$ to the appropriate models, the junction temperature is in general lower than in natural convection model. However, the first model results in a maximum temperature in this simulation, whereas the results of the adhesive based models results in the lowest junction temperature.

Table 6: Forced Convection Junction Temperature Results

\begin{tabular}{|c|c|}
\hline No. & $\mathrm{T}_{\mathrm{j}}$ \\
\hline & ${ }^{\circ} \mathrm{C}$ \\
\hline 2 & 105.9 \\
\hline 4 & 105.8 \\
\hline 6 & 104.8 \\
\hline
\end{tabular}

\section{Conclusion}

In this paper, six PCB models have been simulated. Three models were simulated with a natural convection, whereas three models were simulated with forced convection. Moreover, the influence of thermal conductivity of dielectric layer in IMS PCBs has been shown and compared to the proposed adhesive based PCBs. The proposed adhesive based PCBs achieved the lowest junction temperature, in both natural convection model and forced convection model. By comparing the initial IMS model with a thermal conductivity of dielectric layer of $3 \mathrm{Wm}^{-1} \mathrm{~K}^{-1}$ with the adhesive based model and a forced convection of $2 \mathrm{~ms}^{-1}$, a maximum $\Delta T_{\max }=6.3^{\circ} \mathrm{C}$ was achieved.

\section{Literature}

[1] H. M. Cho and H. J. Kim, "Metal-Core Printed Circuit Board With Alumina Layer by Aerosol Deposition Process," IEEE Electron Device Letters, vol. 29, no. 9, pp. 991-993, Sep. 2008.

[2] M. J. Yoo, H. M. Cho, S. H. Kim, H. S. Lim, S. D. Park, W. S. Lee, J. S. Kim, T. G. Seong, B. J. Jeong and S. Nahm, "Electric Field Assembled Anisotropic Dielectric Layer for Metal Core Printed Circuit Boards," IEEE Electron Device Letters, vol. 33, no. 11, pp. 1607-1609, Nov. 2012.

[3] S. C. Shen, H. J. Huang, and H. J. Shaw, "Design and Estimation of a MCPCB-Flat Plate Heat Pipe for LED Array Module," in Mechatronics and Automation, pp. 158-163, Aug. 2013.

[4] E. Juntunen, O. Tapaninen, A. Sitomaniemi, M. Jämsä, V. Heikkinen, M. Karppinen and P. Karioja, "CopperCore MCPCB With Thermal Vias for High-Power COB LED Modules," IEEE Transactions on Power Electronics, vol. 29, no. 3, pp. 1410-1417, Mar. 2014.

[5] V. Heikkinen, E. Juntunen, K. Kautio, A. Kemppainen, P. Korhonen, J. Ollila, A. Sitomaniemi, T. Kemppainen, H. Korkala, T. Kutilainen and $H$. Sahavirta, "High-brightness RGB LED Modules Based on Alumina Substrate," Vtt Fi, pp. 672-677, June 2007.

[6] S. Klarmann, B. Manesh, T. Hoenle and Y. Vagapov, "Analysis of Insulated-Metal-Substrates Structures in the Context of Heat Dissipation Enhancement," Internet Technologies and Applications, Sep. 2017.

[7] S. Di Pascoli, P. E. Bagnoli, and C. Casarosa, "Thermal Analysis of Insulated Metal Substrates for Automotive Electronic Assemblies," Microelectronics journal, vol. 30, no. 11, pp. 1129-1135, Nov. 1999.

[8] J. Lee, H. Shin, J. Kim, and H. Yoon, "Filler Effect of Low Loss Dielectricity in Printed Circuit Board Material," Journal of Electroceramics, vol. 23, no. 2-4, pp. 141-145, Oct. 2009.

[9] H. Zhang, S. Xu, H. Chen, and T. Lin, "Thermal Analysis and Characterization of Electronic Packages with Alternative Lid Coatings," in Electronic Packaging Technology, pp. 342-347, Aug. 2017.

[10] T. R. Ogden, Thermal Conductivity of Hard Anodized Coatings on Aluminum, Naval Ocean Systems Center San Diego, CA, 1987.

[11] R. C. Agarwala and V. Agarwala, "Electroless alloy/composite coatings: A review," Sadhana, vol. 28, no. 3-4, pp. 475-493, June 2003.

[12] X. C. Tong, Advanced materials for thermal management of electronic packaging. New York, NY: Springer, 2011. 